\title{
Hoechst AG gegen Arbeitsgericht Frankfurt am Main - Eine Dokumentation
}

In ihrer kurzen Geschichte stand die Arbeitsgerichtsbarkeit häufig im Mittelpun] politischer und wissenschaftlicher Kontroversen. Als Institution, die den grundl genden gesellschaftlichen Konflikt zwischen Arbeit und Kapital befrieden und dam beherrschbar machen soll, sieht sie sich per Konstruktion der ständigen Kontrol und Einflußnahme der sozialen Widersacher ausgesetzt. Damit die Arbeitsgerich ihre gesellschaftlichen Integrationsaufgaben im Spannungsfeld von Kapital ur Arbeit wahrnehmen können, hat der Gesetzgeber ihre Zusammensetzung in ein Weise geregelt, die fundamental von der anderer Gerichte abweicht. In allen Instat zen der Arbeitsgerichtsbarkeit werden die Berufsrichter von ehrenamtlichen Ricl tern ergänzt, die die gegensätzlichen Interessen von Gewerkschaften und Unternel mern personell repräsentieren. Freilich birgt die »Institutionalisierung des Klasseı konflikts«, für welche die Arbeitsgerichtsbarkeit ein anschauliches Beispiel darstell stets auch Tendenzen in sich, die zu ihrer Auflösung drängen. Denn die Verallg, meinerung antagonistischer Interessen kann niemals ein Modell gesellschaftlich Konfliktverarbeitung hervorbringen, das im Wechsel ökonomischer Phasen daue hafte Lösungen ermöglicht. Das Niveau und die Intensität gesellschaftlicher Kor flikte drängen deshalb mit einer gewissen zeitlichen Verzögerung auch in $d$ Arbeitsgerichte und bestimmen deren Formen der Auseinandersetzung.

Wenn die Arbeitsgerichtsbarkeit Justiz zur Regelung des gesellschaftlichen Grunc konflikts ist, dann sind die ökonomischen Rahmenbedingungen sowie die gesel schaftlichen Wertvorstellungen der Richter Bedingungen dafür, welchen Weg d: Rechtsprechung einschlägt. Otto Kahn-Freund' hat für die Weimarer Republi gezeigt, daß eine konservative Richterschaft auf die wechselhafte Wirtschaftsen wicklung in den zwanziger Jahren mit faschistischen Ordnungsmodellen (entspr، chend dem Italien Mussolinis) reagiert hat. Eine im wesentlichen konservativ orier. tierte Arbeitsgerichtsbarkeit prägte die Entwicklung der Bundesrepublik vom vol ständigen wirtschaftlichen Zusammenbruch 1945 bis zum Wiederaufstieg als füt rende Industrienation. ${ }^{2}$ Unbekannt in der Geschichte der Arbeitsgerichtsbarke hingegen ist ein Konfliktszenario, in dem eine ökonomische Depression und Ansatz punkte einer fortschrittlichen, die Interessen von Arbeitnehmern stärker berücksick tigenden Rechtsprechung aufeinandertreffen. Seit Ende der siebziger Jahre finden i der Arbeitsgerichtsbarkeit Auseinandersetzungen vor allem zwischen erstinstanzl: chen und übergeordneten Gerichten statt, in denen typische Probleme der Bewält: gung ökonomischer Krisenfolgen zur Debatte stehen. Wenn Arbeitsgerichte in Fra

\footnotetext{
I Das soziale Ideal des Reichsarbeitsgerichts, in: Th. Ramm (Hg.), Arbeitsrecht und Politik. Quellentex 1918-1933, Neuwied am Rhein, Berlin-Spandau 1966, S. 149 ff.

2 W. Däubler, Das soziale Ideal des Bundesarbeitsgerichts, in: M. Kittner (Hg.), Streik und Aussperrun Frankfurt am Main und Köln I974, S. 4II ff.
} 
gen wie der Zulässigkeit der Aussperrung ${ }^{3}$, der Existenz eines Weiterbeschäftigungsanspruchs nach der Kündigung ${ }^{4}$ und des Schutzes von Schwangeren Urteile gefällt haben, die entscheidend von dem abweichen, was das bisherige "Sozialideal« der Arbeitsgerichtsbarkeit ausmachte, dann liegt das nicht zuletzt daran, daß sich erstmals in der deutschen Geschichte dieser Gerichtszweig gegenüber dem Sozialstaatsgrundsatz verpflichteten Richtern geöffnet hat.

Solange sich die Konfliktfronten im Rahmen der gesellschaftlich akzeptierten Institutionen von Kapital und Arbeit hielten, konnte sich eine neue arbeitsgerichtliche Rechtsprechung entwickeln, die für eine (ihrem Anspruch nach) demokratisch-pluralistische Gesellschaft angemessen ist. In allen wichtigen Fällen entschieden die Arbeitsrichter im argumentativen Schutz der Gewerkschaften, die sich seit Ende der sechziger Jahre für Veränderungen eines Teils der Rechtsprechung eingesetzt hatten. ${ }^{6}$ Im Kontext vorsichtiger Annäherung zwischen Teilen der Arbeitsgerichtsbarkeit und den Gewerkschaften entstanden Kooperationsformen, zu denen als bedeutsamste das gewerkschaftliche Engagement von Arbeitsrichtern gehört. Zum anfänglich bestaunten, sodann gewohnten politischen Alltag wurde es alsbald, daß gewerkschaftlich organisierte Richter ihre Meinung öffentlich bekundeten: gegen den Bau der Frankfurter Startbahn West, die Aufstellung amerikanischer Mittelstreckenraketen und zu anderen, weithin umstrittenen Themen.

All dies war freilich nur unter der Voraussetzung möglich, $d a ß$ in Konfliktfällen mit dem gesellschaftlichen Widerpart, den Unternehmern, die Gewerkschaften für ihre Mitglieder, die Richter, eintraten. Es dürfte nicht zufällig sein, daß Unternehmer, die diesem experimentierfreudigen Treiben in der Arbeitsgerichtsbarkeit ein Ende setzen wollten, einen Fall zum Konflikt stilisierten, in dem die Fronten verschoben waren, in dem die Gewerkschaft dem Arbeitsgericht den argumentativen Schutz versagte oder mit so wenig Nachdruck vertrat, daß eine öffentlich vorgetragene Kritik der Unternehmer erfolgreich sein konnte. Der nachfolgend dokumentierte Fall Krauss gegen Hoechst AG, der zu einem Fall Hoechst AG gegen Arbeitsgericht Frankfurt am Main wurde, scheint für das betroffene Unternehmen und die seine Interessen öffentlich vortragende Presse diesen exemplarischen Charakter zu besitzen.

Was war geschehen? Im Dezember 198 I hatte die Hoechst AG ein Betriebsratsmitglied mit Zustimmung des Betriebsrats gekündigt, weil dieses seinen Arbeitgeber verleumdet und beleidigt habe. Die öffentlich vorgetragene Äußerung, die Hoechst AG »sei schon jahrelang in der Lage, die Hessische Landesregierung mit der Drohung des Verlustes von Arbeitsplätzen zu erpressen«, berechtige den Arbeitgeber zur fristlosen Kündigung eines Arbeitnehmers. Um die Dynamik dieses scheinbar alltäglichen Falles zu verstehen, muß man wissen, daß der Gekündigte Mitglied einer alternativen linken Liste bei der Betriebsratswahl war, die es sich zum Ziel gesetzt hatte, die Politik des seit Jahren amtierenden konservativen Betriebsratsvorsitzenden durch stärker konfliktorientierte Aktionen der Belegschaft abzulösen. An der Kündigung hatte infolgedessen nicht nur der Arbeitgeber, sondern auch der Betriebsrat und - da der Betriebsratsvorsitzende als Hauptvorstandsmitglied der IG Chemie, Papier, Keramik der vorherrschenden konservativen Mehrheit angehört - die Gewerkschaft ein Interesse. Das für die Klage des Entlassenen zuständige Arbeitsgericht Frankfurt war - und hiermit nimmt die Geschichte ihren für die deutsche

\footnotetext{
3 R. Erd, Entwicklungstendenzen im Aussperrungsrecht - eine Dokumentation, Kritische Justiz 1978, S. $404 \mathrm{ff}$.

4 R. Erd, Gesetzlicher Kündigungsschutz und Wirtschaftskrise, Kritische Justiz 1982, S. ${ }_{3} 67 \mathrm{ff}$.

5 Kritische Justiz 1982 , S. 419 ff.

6 U. Zachert, M. Metzke, W. Hamer, Die Aussperrung, Köln 1978
} 
Arbeitsgerichtsbarkeit untypischen Verlauf - nicht der Ansicht des Arbeitgebers und erklärte im Mai 1982 die Kündigung für unwirksam. ${ }^{7}$ Der argumentative Weg zu diesem Urteil ist alles andere als radikal, wie vielfach in den nun folgenden öffentlichen Kontroversen suggeriert wurde. Das Arbeitsgericht rekurriert auf die etablierte Rechtsprechung des Bundesverfassungsgerichts zur Meinungsfreiheit (Lüth-Urteil BVerfGE 7, S. 198 ff.) und befindet, daß eine Güterabwägung zwischen der Meinungsfreiheit (Art. , GG) und den Strafrechtstatbeständen der $\$ 185 \mathrm{ff}$. StGB stattzufinden habe. In außerordentlich differenzierter Weise begründet es sodann, daß im konkreten Falle die Grundrechtsausübung durch den Kläger keinen Verstoß gegen die Grundregeln des Arbeitsverhältnisses darstelle.

Der Versuch, der Meinungsfreiheit im autoritär strukturierten kapitalistischen Arbeitsprozeß punktuell zum Durchbruch zu verhelfen, mußte freilich diejenigen provozieren, die an der Aufrechterhaltung solcher Verhältnisse ein materielles Interesse haben. Die Frankfurter Allgemeine Zeitung machte sich zum Sprecher einer Kritik am Arbeitsgericht Frankfurt, die zunächst nur mit den Mitteln der Ranküne arbeitete und dabei dem betroffenen Richter noch eine gewisse Sympathie entgegenbrachte (»Barockengel «) $)^{8}$, bald jedoch sich zu einer Form steigerte, die ihre Absicht unumwunden preisgab: Einflußnahme auf die Arbeitsgerichtsbarkeit, um eine Rechtsprechung zu revidieren, die für eine stärkere Geltung von politischen Grundrechtsgarantien im Arbeitsverhältnis eintritt.

Um dies zu erreichen, bedurfte es freilich eines weiteren Akteurs, denn auch ein mächtiges Unternehmen und eine ihm wohlgesonnene Presse kennen die Grenzen ihrer Macht. In den Boden, den die FAZ so fruchtbar gemacht hatte, pflanzte die Hoechst AG nun ein mit wissenschaftlicher Weihe versehenes Rechtsgutachten ein, das Zweifel daran, die bisherigen Akteure hätten nur ihre Interessen vertreten, beseitigen sollte. Professor Dr. Friedrich Geerds von der Universität Frankfurt, der übrigens dem Autor dieses Beitrags vor 15 Jahren noch kritische Einsichten in das Strafrecht vermittelte, schlüpfte in die Rolle des wohl nicht schlecht dotierten Hilfsorgans und begründete auf 56 Seiten einen Vorwurf, der so waghalsig ist, daß nur die allersorgfältigste Arbeit den tiefen Sturz ihres Urhebers verhindern kann. Rechtsbeugung, ein gegen die grausamsten Nazi-Richter nur äußerst selten erhobener Vorwurf ${ }^{9}$, meint Professor Geerds dem Richter nachweisen zu können, der sich für die grundgesetzlich geschützten Interessen eines Arbeitnehmers engagiert hatte.

Wie nun fädelte die Hoechst AG das Geerds'sche Gutachten, das die Aussichten für ein Strafverfahren wegen Rechtsbeugung gegen den betroffenen Richter für günstig erachtet, in den arbeitsgerichtlichen Entscheidungsprozeß ein? Auf durchaus ungewöhnliche, die Absicht deutlich erkennbar lassende Weise. Die Hoechst AG, seit Mai 1983 im Besitz des Gutachtens, erhob nicht etwa, das wäre der übliche Weg, Strafanzeige wegen Rechtsbeugung, sondern gab Geerds' Auftragsarbeit zu den Akten eines anderen Verfahrens, das an der Kammer von Richter Feser anhängig war, sowie in das Berufungsverfahren gegen das erstinstanzliche Urteil. »Wir wollten Richter Feser und dem Präsidenten des Landesarbeitsgerichts Gelegenheit geben, die Angelegenheit intern zu regeln«, erklärte der Rechtsvertreter der Hoechst AG diesen Schritt (Frankfurter Rundschau vom 6.9. 1983). Und in einem Brief vom 5. 9. 1983 an den Präsidenten des Landesarbeitsgerichts Frankfurt, Dr. Fischer, und den Arbeitsgerichtsdirektor, Jürgen Schuldt, forderte das Unternehmen, "gebotene Maßnahmen« zu treffen, bevor es sich gezwungen sehe, die Staatsanwaltschaft ein-

7 Az. ${ }_{2}$ Ca $31 / 82$; teilweise abgedruckt in: Kritische Justiz 1983, S. 69 ff.

8 Vgl. dazu die Anmerkung von U. Mückenberger zum Urteil der I2. Kammer des Arbeitsgerichts Frankfurt in: Kritische Justiz 1983 , S. 8 I ff.

9 Dazu in Heft $2 / 1984$ der Kritischen Justiz der Aufsatz von Ingo Müller. 
zuschalten (Frankfurter Allgemeine Zeitung vom 7. 9. 1983). Eine Dienstaufsichtsbeschwerde gegen Richter Feser im Kontext eines anderen Rechtsstreits rundete den juristischen Angriff der Hoechst AG ab.

Über die Aufgaben seines Rechtsgutachtens schreibt Friedrich Geerds:

"Dieses Rechtsgutachten erstatte ich für die Hoechst AG zu der Frage, ob die Leitung und Entscheidung in dem vom Arbeitsgericht Frankfurt a. M. am I I. Mai 1982 entschiedenen Rechtsstreit (Az.: ... Ca 31/82) durch den Vorsitzenden Richter am Arbeitsgericht Frankfurt

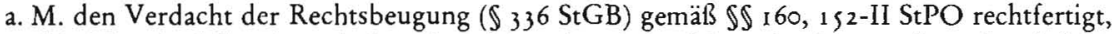
und bejahendenfalls, ob nach dem derzeitigen Stand der Dinge damit zu rechnen ist, daß die Staatsanwaltschaft die Voraussetzungen von $\$ 170-\mathrm{I}$ StPO für gegeben erachten würde $[\ldots]$

Trotz der sich z. T. durch die für ein Strafverfahren über Rechtsbeugung wesentlichen Begriffe der Leitung und Entscheidung in einer Rechtssache ergebenden tatsächlichen Zusammenhänge ist die arbeitsrechtliche Beurteilung dieses Rechtsstreits über die Wirksamkeit einer Kündigung aus wichtigem Grunde allein Sache der dafür zuständigen Arbeitsgerichte und nicht Gegenstand dieses Gutachtens. [...] Für den hier zu prüfenden Tatverdacht der Rechtsbeugung $(\$ 336 \mathrm{StGB})$ würde eine Befangenheit des Vorsitzenden Richters allein nicht genügen, sondern müssen tatsächliche Anhaltspunkte dafür vorliegen, daß er sich bei Leitung oder Entscheidung dieser Rechtssache einer vorsätzlichen Beugung des Rechts zugunsten oder zum Nachteil einer Partei schuldig gemacht hat. Es kommt für dieses Gutachten mithin allein auf diejenigen Tatsachen an, die für die strafprozessuale Beurteilung der mir gestellten Fragen und dafür wesentlichen Vorfragen strafrechtlicher Art angesichts der prozessualen Handhabung und Entscheidung dieser Rechtssache als bedeutsam erscheinen.

Im folgenden ist zwischen den Voraussetzungen der Einleitung eines Strafverfahrens [.. .] und der nach dem derzeitigen Stand meines Materials wahrscheinlich zu erwartenden Entscheidung der Staatsanwaltschaft über die Erhebung der Anklage [.. . zu unterscheiden, um der Hoechst AG Gelegenheit zu geben, sich über die in Frage stehende Strafanzeige schlüssig zu werden.«

Unter der Überschrift " $Z$ u den für eine Strafanzeige wichtigen Voraussetzungen der Einleitung eines Strafverfahrens« schreibt Geerds u. a.:

"Als tatbestandsmäßiges Handeln ist in $\$ 336 \mathrm{StGB}$ ein Verhalten beschrieben, durch das der Richter bei Leitung oder Entscheidung einer solchen Rechtssache vorsätzlich ( $\$$ I S StGB) das Recht zugunsten oder zum Nachteil einer Partei beugt. Eine derartige Rechtsbeugung kann zum einen bei der Prozeßleitung oder der Entscheidung und zum anderen im Hinblick sowohl auf das für den fraglichen Rechtsstreit maßgebende materielłe Recht als auch für das zur Verwirklichung desselben wichtige Verfahrensrecht begangen werden [...]

Bevor ich auf eine ganze Reihe von Punkten eingehe, die trotz der soeben angedeuteten Schwierigkeiten im Verfahren vor dem Arbeitsgericht Frankfurt a. M. und in seinem Urteil meiner Ansicht nach zumindest den Verdacht der parteilichen, falschen Rechtsanwendung erwecken können und deshalb als zureichende tatsächliche Anhaltspunkte im Sinne des $\$$ I $52-$ II StPO in Betracht zu ziehen sind, sei kurz noch etwas zur subjektiven Seite strafrechtlicher Verantwortlichkeit gesagt; für diese reicht seit der Änderung des $\$ 336 \mathrm{StGB}$ durch das EGStGB vom 2. 3. I 974 nach ganz überwiegend vertretener Rechtsansicht vorsätzliches Handeln im Sinne des Dolus eventualis aus. Dafür aber genügt, trotz in Nuancen divergierender Vorsatzlehren, wenn der Täter zumindest mit einer falschen, für eine der Parteien des Rechtsstreits nachteiligen - somit die andere Partei begünstigenden - Rechtsanwendung rechnen und diese für möglich halten mußte und dies willensmäßig gebilligt hat. Im Gegensatz zur alten Fassung ist subjektiv weder sicheres Wissen um die falsche Rechtsanwendung bzw. die Nachteile für eine Partei noch gar eine entsprechende Absicht oder Motivation erforderlich [...] Bei einer Vielzahl z. T. grober Rechtsfehler bleibt für meine Begriffe in dieser Sache für die subjektive Seite nur die Alternative zwischen einem Falle ungewöhnlicher, wenngleich unvorsätzlicher Ignoranz und dolosem Handeln. Deshalb ist m. E. im konkreten Falle vorsätzliches Handeln zumindest nicht so sicher auszuschließen, daß man den einfachen Tatverdacht verneinen kann. Vielmehr erscheinen mir gerade insoweit umfassende und gründliche Ermittlungen der Staatsanwaltschaft als angebracht. « (Hervorhebung von mir, R. E.)

Geerds untersucht nun - das soll hier nicht dokumentiert werden - eine Reihe von angeblichen prozessualen Fehlern, wie dies typischerweise Berufungsgerichte tun, ohne deshalb freilich der untergeordneten Instanz Rechtsbeugung vorzuwerfen. So 
hält er dem Vorsitzenden Richter beispielsweise vor, dieser habe die Entscheidungserheblichkeit eines Bew eismittels falsch eingeschätzt. Würde das, was Geerds an dem Urteil des Arbeitsgericht Frankfurt kritisiert (einmal unterstellt die Kritik wäre zutreffend), bereits den Verdacht auf Rechtsbeugung begründen, so müßte ein Großteil der erst- und zweitinstanzlichen Richter fürchten, alsbald wegen Rechtsbeugung verurteilt zu werden. Doch allein auf der Ebene prozessualer Fehler, das merkt auch Geerds, kann er seine schwerwiegende Behauptung nicht begründen, ohne ein Schmunzeln der Rechtswissenschaft und -praxis zu provozieren. Er lenkt seine Argumentation deshalb in eine zweite Richtung, die er doch seinem Anspruch nach gerade nicht einschlagen wollte. Hatte er zu Beginn selbstbeschränkend formuliert, daß die arbeitsrechtliche Beurteilung des Rechtsstreits seine Aufgabe nicht sei, so begibt er sich nun genau auf dieses Gebiet und präsentiert eine zum Arbeitsgericht Frankfurt alternative Interpretation der $\$ \mathbb{S}$ i 8 ff. StGB und Art. $5 \mathrm{GG}$. Der auf schwachen Argumenten stehende Vorwurf der Rechtsbeugung soll dadurch untermauert werden, daß Geerds dem Arbeitsgericht eine unrichtige Norminterpretation unterstellt.

"[...] Gegen die von mir aufgezeigte strafrechtliche Qualifizierung der Äußerungen des Klägers in Sossenheim und ihrer Veröffentlichung in der 'Frankfurter Rundschau< als üble Nachrede oder Belejdigung könnte nach allem allein sprechen, daß sich der Kläger insoweit auf einen Unrechtsausschließungsgrund berufen kann. Als solche kommen bei Ehrverletzungen insbesondere die Wahrnehmung berechtigter Interessen $(\$ 193 \mathrm{StGB})$ sowie das selbstverständlich schon dabei zu berücksichtigende Grundrecht der freien Meinungsäußerung (Art. s GG) in Betracht. Mit diesem hat sich das Arbeitsgericht in seinem Urteil sehr ausführlich befaßt. Es gelangt - u. a. in seiner später noch zu behandelnden kritischen Auseinandersetzung mit der Rechtsprechung des Bundesarbeitsgerichts - abschließend zu dem Ergebnis, daß bei Konzedieren spolemischer Schärfer das Verhalten des Klägers als bloße Meinungsäußerung gerechtfertigt sei und auch der Schrankenvorbehalt des Artikels s-II GG hier nicht eingreife.

Auch diese für die arbeitsgerichtliche Entscheidung m. E. wesentliche Argumentation ist strafrechtlich aus den zuvor dargelegten Gründen und insbesondere hinsichtlich der Beurteilung der Rechtswidrigkeit keineswegs zwingend und zweifelsfrei, sondern legt meiner Ansicht nach zumindest den einfachen Tatverdacht einer falschen Rechtsanwendung im Sinne des $\$ 336$ StGB nahe. Dabei kann hier dahingestellt bleiben, wie bei Verneinen einer Verleumdung ( $\$$ i 87 $\mathrm{StGB})$ in Fällen übler Nachrede $(\$ 186 \mathrm{StGB})$ oder Beleidigung $(\$$ I $85 \mathrm{StGB})$ die Grenzen des Art. 5 GG im einzelnen zu beurteilen und welche arbeitsrechtlichen Konsequenzen daraus zu ziehen sind. Denn ausschlaggebend für die strafprozessuale Problematik ist allein, daß die im arbeitsgerichtlichen Urteil in diesem Zusammenhang gemachten Ausführungen zum einen strafrechtlich und verfassungsrechtlich fehlsam sein können und zum anderen den Verdacht einer prozessual falschen Entscheidungsfindung zu begründen vermögen, die in dieser Sache eindeutig zu Lasten der Beklagten gehen würde. Denn mit der vom Arbeitsgericht bejahten Rechtfertigung wird ein für die von der Beklagten ausgesprochene fristlose Kündigung wichtiger, deshalb zuerst geltend gemachter Grund rechtlich irrelevant. Im einzelnen stütze ich mich hier für den Verdacht einer falschen Recbtsanwendung (Hervorhebung von mir, R. E.) durch das Arbeitsgericht und seinen Vorsitzenden Richter auf folgende Punkte seiner Urteilsbegründung.

(a) Das Arbeitsgericht läßt die Frage dahinstehen, ob die Äußerungen des Klägers als Werturteile oder als 'Tatsachenbehauptungen anzusehen sind, weil in jedem Falle Art. s GG zu beachten sei. Diese Argumentation ist strafrechtlich selbst dann nicht haltbar, wenn man eine Verleumdung verneint, weil die Grenzen des Rechts der freien Meinungsäußerung auch in Fällen übler Nachrede $(\$ 186 \mathrm{StGB})$ und Beleidigung divergieren könnten und zu beachten sind. Das übrigens auch solche Ehrverletzungen bei einer fristlosen Kündigung arbeitsrechtlich bedeutsam sein können, hat zudem - wie das Urteil selbst ausweist - das Bundesarbeitsgericht zumindest prinzipiell anerkannt, wenngleich Differenzierungen der genannten Art - soweit mir ersichtlich - zu vermissen sind. Zumindest aber ist das Unterlassen dieser Differenzierung von Werturteil und Tatsachenbehauptung insoweit eine möglicherweise falsche Rechtsanwendung (Hervorhebung von mir, R. E.) zu ungunsten der Beklagten.

(b) Zutreffend an die Rechtsprechung des Bundesverfassungsgerichts anknüpfend geht das Arbeitsgericht davon aus, daß der Gesetzeswortlaut des Art. 5-II GG nicht als einseitige Beschränkung eines Grundrechts durch allgemeine Gesetze - hier z. B. die $\$ \$ 186$, I85 StGB - 
aufzufassen sei, sondern eine konkrete Abwägung der kollidierenden Rechtsgüter zu erfolgen habe. Diese nimmt es dann nach der in anderem Zusammenhang noch zu würdigenden kritischen Auseinandersetzung mit der einschlägigen Judikatur des Bundesarbeitsgerichts mit dem Bemerken vor, daß diese nicht unbesehen übernommen werden könne. Die dann vom Arbeitsgericht vorgenommene Güterabwägung aber geschieht in $\mathrm{m}$. E. rechtlich anfechtbarer Weise, weshalb mir auch insoweit zumindest der Verdacht einer falschen Rechtsanwendung zum Nachteil der Beklagten als begründet erscheint $[\ldots]$

(c) Das arbeitsgerichtliche Urteil, das in den fraglichen Äußerungen des Klägers auch keine Verletzung seiner arbeitsvertraglichen Pflichten erblickt, argumentiert meiner Ansicht nach auch in diesem Zusammenhang anfechtbar und einseitig zu Lasten der Beklagten [...]

Anfechtbar und den Verdacht falscher Rechtsanwendung erweckend ist es $\mathrm{m}$. E. jedoch, wenn das Arbeitsgericht sich in diesem Zusammenhang pauschal auf die Freiheit der MeinungsäuBerung, d. h. auf Art. s GG, beruft. Das Urteil verkennt, wenn es auf die anscheinend allein von ihm zu beurteilende "politische Auseinandersetzung « sowie das dabei Übliche und Zulässige abstellt, sowohl die Gefahren eines dann möglichen, rechtlich unzulässigen Mißbrauchs dieser Qualifikation - und damit eines Grundrechts - als auch die trotz der erforderlichen konkreten Güterabwägung bestehenden Grenzen und damit Art. s-II GG. Diese bedenkliche, rechtlich nicht haltbare Einseitigkeit wird nicht dadurch richtiger, daß das Gericht meint, sich insoweit darauf stützen zu können, die Beklagte habe den politischen Charakter der Äußerung des Klägers anerkannt. Seine alles auf die politische Auseinandersetzung abstellende, die Grenzen des Art. s-II GG im wesentlichen ignorierende Argumentation muß überdies aus folgenden Gründen als anfechtbar und in dieser ungebührlichen Verallgemeinerung als falsch angesehen werden. Wäre dieser Recbtsstandpunkt des Arbeitsgerichts (Hervorhebung von mir, R. E.) richtig, so bedürfte es kaum solcher Verfassungsvorschriften wie der des Art. 46-I GG für Abgeordnete des Deutschen Bundestages über Straflosigkeit (Indemnität) wegen gewisser Ehrverletzungen, wobei durch Art. 46-I, S. 2 sogar noch Verleumdungen ( $\$ 187 \mathrm{StGB}$ ) ausgenommen sind. Können sich mithin nicht einmal Abgeordnete bei Äußerungen in Bundes- oder auch Länderparlamenten bzw. deren Ausschüssen für Ehrverletzungen insoweit schlicht auf eine Argumentation wie die vom Arbeitsgericht Frankfurt a. M. praktizierte berufen, so dürfte sich dieses bei anderen politischen Auseinandersetzungen erst recht nicht in dieser Weise über ersichtlich bestehende Grenzen des Grundrechts der freien Meinungsäußerung hinwegsetzen, womit sich - m. E. fälschlich - Straftaten rechtfertigen ließen. Die Rechtsausführungen im arbeitsgerichtlichen Urteil können ferner auch deshalb nicht richtig sein, weil strafrechtlichen Sonderregelungen wie der des $\ 187$ a StGB gegen sog. politische Beleidigungen dann tatsächlich weithin der Boden entzogen wäre. Ebenso wie derartige Verhaltensweisen von Berufspolitikern, auch Äußerungen von Abgeordneten außerhalb der Parlamente, könnten wie Journalisten oder Redakteure sich auch politisch engagierte Bürger ohne weiteres auf die vom Arbeitsgericht Frankfurt a. M. für richtig gehaltenen Grundsätze berufen, um tatbestandsloses oder rechtmäßiges Handeln bescheinigt zu bekommen. Das aber kann für Verstöße gegen $\ 187 \mathrm{a}$ StGB selbst dann nicht richtig sein, wenn man hier bei der Ehrenrührigkeit die politischen Verhältnisse in gebührender Weise berücksichtigt. - Auch diese insoweit m. E. falsche Rechtsanwendung konnte ersichtliche für die Beklagte nachteilige Konsequenzen bei der Entscheidung dieser Rechtssache haben $[\ldots]$

(e) Insgesamt erweckt diese Entscheidung des Arbeitsgerichts - an seiner Begründung gemessen - bezüglich dieser Vorgänge in Sossenheim und des sich darauf beziehenden Presseberichts sowohl in der prozessualen Handhabung als auch im Hinblick auf strafrechtliche Vorfragen $\left(\$ \mathrm{I}_{1}\right.$, ff. StGB) und Art. $5 \mathrm{GG}$ den Eindruck einer trotz aller Ausführlichkeit in der Sache oberflächlichen wie einseitigen Argumentation, die deshalb den Verdacht der falschen Rechtsanwendung begründet. Diese kann daher für $\$ 336 \mathrm{StGB}$ relevant sein, weil angesichts der vielen Punkte bei dem für die Urteilsbegründung verantwortlichen Vorsitzenden Richter der in den $\iint$ I $60-I$, I $52-I I$ StPO vorausgesetzte einfache Tatverdacht so $m$. E. auch subjektiv nicht sicher auszuscbließen ist. (Hervorhebung von mir, R. E.) [...]

gg) Zusammenfassend ergibt das fragliche Verfahren vor dem Arbeitsgericht Frankfurt a. M. als die für $\$ 336 \mathrm{StGB}$ hier wesentliche Rechtssache im Zusammenhang mit der schriftlichen Urteilsbegründung meiner Ansicht nach so zahlreiche und z. T. gewichtige tatsächliche Anhaitspunkte für eine der Beklagten zum Nachteil gereichende falsche Rechtsanwendung, daß mir der einfache Tatverdacht ( $\$ \$$ 160-I, I 52 -II StPO) einer Rechtsbeugung ( $\$ 336 \mathrm{StGB}$ ) als begründet erscheint. Denn von der für meine Begriffe unangemessen punktuellen Behandlung der für dieses Verfahren wesentlichen Tat- und Rechtsfragen - hier insbesondere die flüchtige und strafrechtlich nicht überzeugende Argumentation zu den $\$ \$ 185 \mathrm{ff}$. StGB - sind m. E. bisher keine Unrechtsausschließungsgründe so sicher, daß sie die Annahme einfachen Tatverdachts ausschließen. Ebenso wie für den im Urteil nur gestreiften $\$ 193$ StGB gilt das - wie 
oben insbesondere unter cc vor (a) ausgeführt - für das im Hinblick auf seine Grenzen meiner Ansicht nach fälschlich weit interpretierte Grundrecht der freien Meinungsäußerung (Art. s GG), mit welchem das in tatsächlicher Hinsicht bagatellisierende Arbeitsgericht letztlich Verhaltensweisen des Klägers zu rechtfertigen sucht.

Sieht man vom unterschiedlichen Gewicht der von mir aufgezeigten Punkte ab, ist dieser Sachverhalt insgesamt betrachtet doch so geartet, daß man hier bedingt vorsätzliches Handeln des Vorsitzenden Richters bei den zahlreichen zum Nachteil der Beklagten gehenden Rechtsfehlern nicht ausscbließen kann. (Hervorhebung von mir, R. E.) Bleibt in Fällen von Rechtsbeugung oft nur die Alternative zwischen so oder so bedingter Ignoranz und dolosem Handeln des Richters, ist jedenfalls hier bei der Vielzahl der teilweise doch recht groben Rechtsfehler ein gezieltes und mithin vorsätzliches Handeln für meine Begriffe als möglich zu erachten, d. h. einfacher Tatverdacht zumindest nicht auszuschließen. Es wird Aufgabe der Staatsanwaltschaft sein, durch umfassende Klärung des Sachverhalts eine fundiertere Beurteilung des fraglichen Verhaltens zu erreichen als mir das aufgrund meines Materials gegenwärtig möglich ist $[\ldots] \cdot$.

Der Versuch der Hoechst AG, vermittels des Geerds'schen Gutachtens Einfluß auf die Geschäftsverteilung des Arbeitsgerichts Frankfurt zu nehmen, traf einen sensiblen Punkt im Selbstverständnis der Richter - ihre Unabhängigkeit. Die bislang allein in der lokalen Presse ausgetragenen Kontroversen über die Entscheidung der 12. Kammer gegen die Hoechst AG entwickelten sich nun zu einem Konflikt zwischen der Frankfurter Arbeitsgerichtsbarkeit und dem Chemie-Konzern, über den die überregionale Presse ausführlich berichtete. Professor Geerds hatte der Auseinandersetzung eine Dimension verliehen, die auch sein Gutachten zum öffentlich diskutierten Fall werden ließ. Indem er eine Interpretation des Verhältnisses von Strafrechtsnormen und Grundrechten zur alleingültigen erklärt, will er eine andere juristische Meinung kriminalisieren. An keiner Stelle seines Gutachtens ist es Geerds gelungen, den Nachweis zu erbringen, daß Richter Feser den objektiven Tatbestand der Rechtsbeugung erfüllt hat. Er weist statt dessen beharrlich nach, daß er anderer Rechtsansicht als der Richter ist und versicht diese juristische Alltäglichkeit sodann mit der strafrechtlichen Wertung der Rechtsbeugung.

Die konzertierte Aktion von Hoechst, FAZ und Geerds, einem kritischen Arbeitsrichter die Stirn zu bieten, schien trotz ihrer Massivität an der Geschlossenheit der Frankfurter Arbeitsrichter abzuprallen. Selbst Teile der Gewerkschaften (jedoch nicht die IG Chemie!) und der SPD, bislang eher zurückhaltend, stellten sich auf die Seite von Richter Feser und forderten den Schutz der richterlichen Unabhängigkeit (Frankfurter Neue Presse vom 7.9. 1983). Mit Datum vom 6. 9. 1983 schrieb der Direktor des Frankfurter Arbeitsgerichts, Jürgen Schuldt, an den Vorstandsvorsitzenden der Hoechst AG:

»Sehr geehrter Herr Prof. Dr. Sammet

Der Vorsitzende der Kammer 12 des Arbeitsgerichts Frankfurt a. M. hat das Präsidium des Gerichts davon in Kenntnis gesetzt, daß im Rahmen eines in seiner Kammer anhängigen Rechtsstreits die Hoechst AG ein von Prof. Dr. Geerds, Universität Frankfurt a. M., in ihrem Auftrag verfaßtes Gutachten eingereicht hat, in dem der Gutachter zu dem Ergebnis gelangt, es bestehe der hinreichende Verdacht, der vorsitzende Richter habe bei der Leitung und Entscheidung des Kündigungsrechtsstreits Krauss ./. Hoechst AG das Recht gebeugt. Noch am gleichen $\mathrm{Tag}$, an dem ihm das Gutachten bekanntgeworden sei, habe ihn ein leitender Mitarbeiter der Hoechst AG angerufen und ihn unter Hinweis auf das Gutachten gefragt, ob er es nicht im Interesse seiner Person und des Ansehens der Rechtspflege für angezeigt halte, sich künftig in allen Verfahren unter Beteiligung der Hoechst AG selbst abzulehnen. Wenn er den Weg der Selbstablehnung nicht beschreite, zwinge er die Hoechst AG, ihn wegen Befangenheit abzulehnen. Darüber hinaus habe der Anrufer vorgeschlagen, daß er das für die Geschäftsverteilung zuständige Präsidium über diesen Sachverhalt unterrichte und als erste vorsorgliche Maßnahme den für den nächsten $\mathrm{T}_{\mathrm{ag}}$ anstehenden Verhandlungstermin mit Beteiligung der Hoechst AG absetze.

Nunmehr, am 5. 9. 1983 , erhielt ich ein Schreiben der Hoechst AG vom gleichen Tag, dem ein ebenfalls vom 5.9. 1983 datierendes Schreiben an den Präsidenten des Landesarbeitsgerichts 
Frankfurt a. M. beigefügt war, mit dem dieser unter Hinweis auf das von Professor Geerds erstattete Gutachten und ein angebliches Fehlverhalten des Vorsitzenden der Kammer 12 im Zusammenhang mit dem Erlaß eines Versäumnisurteils gegen die Hoechst AG am 2.9. 1983 aufgefordert wird, >die gebotenen Maßnahmen zu treffen, bevor Hoechst AG gezwungen werde, die Staatsanwaltschaft einzuschalten. An das Präsidium des Arbeitsgerichts erging die Bitte zu prüfen, ob der in dem Schreiben an den Präsidenten des Landesarbeitsgerichts geschilderte Vorgang eine Änderung der geschäftsplanmäßigen Zuständigkeit erforderlich mache.

Namens des Präsidiums des Arbeitsgerichts Frankfurt a. M. verwahre ich mich entschieden gegen diesen unerhörten Versuch der Firma Hoechst AG, Einfluß auf die Geschäftsverteilung zu nehmen. Der Umstand, daß ein Professor für Strafrecht und Strafprozeßrecht in einem über soseitigen Gutachten ausbreitet, es sei wahrscheinlich, daß die Staatsanwaltschaft gegen den Vorsitzenden der Kammer I 2 beim Arbeitsgericht Frankfurt a. M. Anklage wegen Rechtsbeugung erheben werde, mag vom Ergebnis her insbesondere für den Laien beeindruckend sein. Jedem fachlich Vorgebildeten wird jedoch schon nach einmaliger Lektüre des Gutachtens auffallen, mit welcher geradezu abenteuerlichen Kühnheit der Gutachter zu der Auffassung gelangt, der Vorsitzende der Kammer 12 habe das Recht bewußt falsch angewendet. Besonders bedenklich erscheint es deshalb, daß unter dem Deckmantel scheinbarer wissenschaftlicher Erhärtung des Verdachts der Rechtsbeugung in bezug auf die Person des Vorsitzenden der Kammer 12 ein Dauerbefangenheitszustand provoziert werden soll. Das Präsidium des Arbeitsgerichts Frankfurt a. M. läßt Sie wissen, daß es nicht gewillt ist, daran mitzuwirken, die $\mathrm{Fa}$. Hoechst AG auf die von ihr in Szene gesetzte Weise dem gesetzlichen Richter zu entziehen.

Mit freundlichen Grüßen Schuldt"

Der Vizepräsident des Landesarbeitsgerichts, Dieter Bergmann, ließ in einem Brief an die Hoechst AG vom 9. 9. 1983 keinen Zweifel daran, daß er ihre Prozeßstrategie mißbilligt.

"Sehr geehrte Herren,

[...] Ich komme nun zu den Vorwürfen, die Sie im Zusammenhang mit dem Rechtsstreit Krauss ./. Hoechst AG - 12 Ca 31/82 - ArbG Ffm gegen den Richter Feser erheben. - Dabei ist zunächst festzuhalten, daß das Urteil der 1 2. Kammer des Arbeitsgerichts Ffm vom 11 . 5. 1982 Ihren Prozeßbevollmächtigten am 2. 9. 1982 zugestellt wurde. Das Rechtsgutachten des Professors Dr. Geerds datiert vom 18. 5. 1983 . In der Zeit bis zum 29. 8.1983 hat Herr Feser in einer Reihe von Arbeitsrechtsstreitigkeiten, in denen die Firma Hoechst AG Partei ist, judiziert, ohne daß dies von Ihnen beanstandet wurde. Nunmehr erheben Sie plötzlich den Vorwurf der Rechtsbeugung gegen Herrn Feser in dem Rechtsstreit Krauss, der längst in der Berufungsinstanz bei dem Landesarbeitsgericht Ffm anhängig ist, und in dem der nächste Verhandlungstermin am 3.10.1983 ansteht. Dieses Vorgehen kann ich nur mit größtem Befremden zur Kenntnis nehmen. Sie wissen, daß der Vorwurf der Rechtsbeugung im Sinne des $\$ 336 \mathrm{StGB}$ der schwerste Vorwurf ist, der gegen einen Richter in bezug auf die Ausübung seines Amtes erhoben werden kann. Er sollte nach meinem Verständnis nur erhoben werden, wenn wirklich schwerwiegende Beweismittel vorhanden sind, die einen hinreichenden Tatverdacht zu begründen vermögen. Einen solchen kann ich nach der Lektüre des Gutachtens nicht erkennen. Ich halte das Ergebnis des Gutachtens für in keiner Weise überzeugend.

Auf der anderen Seite muß die plötzliche Einreichung des Gutachtens Ende August 1983 verbunden mit der telefonischen Androhung der Erstattung einer Strafanzeige gegen den betroffenen Richter und der Anfrage, ob er sich selbst ablehnen wolle, den Verdacht aufkommen lassen, daß mit diesem Vorgehen erreicht werden sollte, für die Zukunft ein weiteres Tätigwerden des Richters Feser in Rechtsstreitigkeiten mit der Firma Hoechst AG auszuschließen. Daß Sie sich einem solchen Verdacht ausgesetzt haben, können Sie aus der Reaktion des Direktors des Arbeitsgerichts Frankfurt am Main namens seines Präsidiums und aus den Berichten in den Frankfurter Tageszeitungen vom 7. und 8. 9. 1983 entnehmen. - Ich bedaure dies außerordentlich! Es ist der erste derartige Fall seit Bestehen der hessischen Arbeitsgerichtsbarkeit, die am 1. 10. 1946 ihre Arbeit aufgenommen hat.

Es kommt aber noch ein weiterer, außerordentlich bedenklicher Vorgang hinzu. Sie haben das Rechtsgutachten des Prof. Dr. Geerds auch dem Vorsitzenden der I I. Kammer des Landesarbeitsgerichts Ffm, in der der Rechtsstreit Krauss ./. Hoechst AG in der Berufungsinstanz anhängig ist, zugeleitet. Wenn Sie nun schreiben, daß >Hoechst auch nur den Anschein vermeiden will, die Rechtsfindung in dem bei der 11. Kammer des Landesarbeitsgerichts anhän- 
gigen Berufungsverfahren durch eine spektakuläre, öffentlichkeitswirksame Strafanzeige bei der Staatsanwaltschaft gegen den Richter, dessen Urteil mit der Berufung angegriffen wird, beeinflussen zu wollen<, so haben Sie dieser Absichtserklärung meines Erachtens dadurch den Boden entzogen, daß Sie das Rechtsgutachten vom 18.5 .1983 in das Berufungsverfahren eingeführt haben. Dies halte ich für eine ebenso außergewöhnliche Aktion wie die Erstattung einer Strafanzeige. Sie setzen sich dadurch der Gefahr aus, in den Verdacht eines Beeinflussungsversuches gegenüber den Berufungsrichtern zu geraten.

Zum Schluß möchte ich darauf hinweisen, daß ich genau wie Sie die Gesamtumstände des Falles mit großer Sorge betrachte. Ich muß es selbstverständlich Ihnen überlassen, ob und welche weiteren Schritte Sie gegen den Richter Feser für geboten halten. Als Inhaber der Dienstaufsicht habe ich jedenfalls, wie gesagt, keine Veranlassung, irgendwelche Maßnahmen gegen Herrn Feser einzuleiten.

In bezug auf den Rechtsstreit Krauss ./. Hoechst AG kann ich nur dem Wunsch Ausdruck verleihen, daß das Berufungsverfahren unbelastet von den Ereignissen der letzten zwei Wochen sachgerecht zu Ende geführt werden kann.

Bei dem erheblichen Interesse, das die Öffentlichkeit durch die drei großen Frankfurter Tageszeitungen an den entstandenen Problemen nimmt, bitte ich um Verständnis, daß ich dieses Schreiben der Öffentlichkeit nicht vorenthalten kann.

$$
\begin{aligned}
& \text { Hochachtungsvoll } \\
& \text { In Vertretung } \\
& \text { Bergmann } \\
& \text { Vizepräsident « }
\end{aligned}
$$

Die Auseinandersetzungen zwischen der Hoechst AG und den Frankfurter Arbeitsrichtern füllten in der Folgezeit die Leserbriefspalten der Tages- und Wochenzeitungen und eskalierten zu einem grundsätzlichen Problem von wirtschaftlicher Macht und richterlicher Unabhängigkeit, das eine größere Zahl von Richtern auch anderer Gerichtszweige zu öffentlichen Stellungnahmen provozierte. So unterzeichneten 4 I Richter des Amtsgerichts, des Landgerichts und des Oberlandesgerichts Frankfurt einen Protestbrief (Frankfurter Allgemeine Zeitung vom 22. 9. 1983), den der Arbeitskreis Verwaltungsrichter der Vereinigung Demokratischer Juristen unterstützte (Presseerklärung vom 22. 9. 1983), die Stuttgarter Fachgruppe Richter und Staatsanwälte der ÖTV kritisierte das Vorgehen der Hoechst AG als »in der Geschichte der Arbeitsgerichtsbarkeit bisher einmalig“ (Der Spiegel Nr. 39/1983). Die Hoechst AG schien angesichts der breiten öffentlichen Reaktionen den Rückzug anzutreten, als sie erklären ließ, sie werde bis zum Abschluß des fraglichen Berufungsverfahrens vor dem Landesarbeitsgericht Frankfurt keine weiteren rechtlichen Schritte gegen Richter Feser unternehmen (Frankfurter Rundschau vom 22. 9. 1983 ).

Damit war der Konflikt freilich keineswegs beendet. Die Frankfurter Allgemeine Zeitung sprang der Hoechst AG zur Seite, die mit dem Rechtsbeugungs-Gutachten erfolglos geblieben war. Fernando Wassner, der die öffentliche Kampagne gegen Richter Feser einst begonnen hatte ${ }^{10}$, löste mit einem Artikel unter der Überschrift "Hessens Arbeitsgerichte haben Schlagseite eine neue Kontroverse aus, in deren Mittelpunkt nicht mehr eine angebliche Rechtsbeugung, sondern das Problem der Meinungsfreiheit und der Parteimitgliedschaft von Richtern stand. Wassner schrieb:

"[...] Das Arbeitsgericht Frankfurt, eines der großen in der Bundesrepublik, arbeitet gegenwärtig mit is Berufsrichtern, denen jeweils ein von den Gewerkschaften und ein von den Arbeitgebern benannter ehrenamtlicher Richter beiseite sitzen. Von diesen is Berufsrichtern, im Rahmen ihrer Kammer das Zünglein an der Waage der Arbeitsjustiz, gehören nach einigermaßen handfesten Zählungen - Parteibücher gelangen nicht zu Richterpersonalakten - elf einschließlich des Arbeitsgerichtsdirektors entweder zur SPD, zu den Grünen, zur Gewerk- 
schaft ÖTV oder zu mehreren dieser Organisationen gleichzeitig. Zumindest einige von ihnen gehören außerdem zu einem Arbeitsrechtskränzchen der ÖTV, dem im übrigen unter anderem auch Rechtsanwälte angehören, die in Arbeitsrechtsstreitigkeiten gewöhnlich Arbeitnehmer vertreten.

Nun könnte man sich damit trösten, daß über einem Arbeitsgericht mit Schlagseite die nächste Instanz wacht, in diesem Fall das Landesarbeitsgericht Frankfurt, zuständig für Rechtsmittel in Arbeitsstreitigkeiten aus einem der großen deutschen Ballungsräume. Das Landesarbeitsgericht Frankfurt hat 13 Berufsrichter. Zehn von ihnen, darunter der Präsident, gehören nach der Beurteilung halbwegs unverdächtiger Kenner entweder der ÖTV oder der SPD oder sogar beiden Organisationen an. In der Arbeitsgerichtsbarkeit werden die Berufsrichter vom Landesarbeitsminister bestellt, in Hessen regiert seit mehr als 30 Jahren eine SPD-Regierung mit einem Arbeitsminister derselben Couleur [...]« (10.9. 1983)

Die Einäugigkeit des Herrn Wassner, der nicht erwähnte, daß das Desaster der deutschen Justizgeschichte darin liegt, daß ein Großteil der Richter auf der politischen Rechten organisiert war, wurde Gegenstand einer Diskussion in den Leserbriefspalten der $\nRightarrow F A Z$.

"Der Bezirksrichterrat bei dem Landesarbeitsgericht Frankfurt am Main als gewähltes Vertreterorgan aller Richter der hessischen Arbeitsgerichtsbarkeit verwahrt sich mit aller Entschiedenheit gegen die Diffamierungen, die in dem Artikel >Hessische Arbeitsgerichte haben Schlagseite (F.A.Z. vom 10. September) enthalten sind. Der Verfasser, Fernando Wassner, stellt dort durch Verknüpfung von Unterstellungen ein Zerrbild der hessischen Arbeitsgerichtsbarkeit vor.

Hierzu nur einige Aspekte: Es beginnt damit, daß wegen der Zugehörigkeit von Richtern zu politischen Parteien und Verbänden nicht nur ohne weiteres deren Einseitigkeit bei ihrer rechtsprechenden Tätigkeit angedeutet wird, sondern gleich eine solche der gesamten hessischen Arbeitsgerichtsbarkeit. Nichts anderes kann ja wohl aus der Überschrift des Artikels geschlossen werden. Richter haben, wie andere Bürger auch, politische Überzeugungen. Richter aller Gerichtszweige und Instanzen und in allen Bundesländern bekennen sich hierzu auch offen durch Mitgliedschaft in entsprechenden Gruppierungen. Dagegen ist in einer repräsentativen Demokratie, in der politische Parteien entscheidend an der politischen Willensbildung mitwirken, nichts einzuwenden. Aus diesen Gegebenheiten aber nun grundsätzlich auf Schlagseite, insbesondere gerade des sich offen bekennenden Richters wie auch seiner Kollegen zu schließen, ist ohne Nachweis im Einzelfall schlichtweg unlogisch und unzulässig. Dabei würde unterstellt, daß Richter generell nicht fähig seien, zwischen Rechtsanwendung im Einzelfall und politischer oder sonstiger Überzeugung zu unterscheiden.

Das tut in dieser Allgemeinheit nicht einmal Fernando Wassner. Was er aber unterstellt, ist, daß er Mitgliedschaft in bestimmten, ihm anscheinend nicht genehmen politischen Gruppierungen für geeignet hält, die Unparteilichkeit von Richtern zu beeinflussen. Hier ist die Schlagseite aber nicht bei der hessischen Arbeitsgerichtsbarkeit, sondern bei Fernando Wassner. Er möchte wohl am liebsten jedem Richter nur eine bestimmte - von wem bestimmte? - politische Orientierung zugestehen [...]

Gerhard Rossmanith, Vorsitzender des Bezirksrichterrats bei dem Landesarbeitsgericht Frankfurt" (FAZ vom 28.9.1983)

Der Viezpräsident des Landesarbeitsgerichts Frankfurt, Bergmann, wandte sich zum zweiten Mal im Verlaufe der Auseinandersetzung an die Öffentlichkeit und schrieb:

"In seinen Artikeln >Hessens Arbeitsgerichte haben Schlagseite (FAZ vom 10. September) und 'Zuerst Richter، (FAZ vom 14. September) hat Fernando Wassner die hessische Arbeitsgerichtsbarkeit derart angegriffen, daß ich dem mit Nachdruck widersprechen muß. Es ist erstaunlich, mit welcher Großzügigkeit der Autor mit Tatsachen umgeht und dann versucht, dem Leser unhaltbare Schlußfolgerungen nahezulegen.

[...] Er behauptet unter Berufung auf einen shalbwegs unverdächtigen Kenner،, daß Io der I3 Berufsrichter am Landesarbeitsgericht entweder der ÖTV oder der SPD oder sogar beiden Organisationen angehören. Abgesehen davon, daß diese Behauptung nicht den Tatsachen entspricht, ist sie deshalb schlimm, weil damit in der Öffentlichkeit der Eindruck erweckt wird, hier habe man es wohl mit einem Gericht zu tun, an dem nicht unabhängige, sondern voreingenommene Richter tätig sind. Dies halte ich für einen durch nichts gerechtfertigten Versuch, Mißtrauen gegen die Richter des Landesarbeitsgerichts zu erzeugen. 
Natürlich gibt es am Landesarbeitsgericht Richter, die Mitglieder politischer Parteien sind, übrigens nicht nur der SPD. Es gibt auch Richter, die in der ÖTV oder im Deutschen Richterbund organisiert sind. Daraus ohne jeden Anlaß Zweifel an der Unabhängigkeit der Richter herzuleiten ist unhaltbar und besorgniserregend. Der Autor weiß genau, daß auch die Präsidenten und andere Richter an den obersten deutschen Gerichten Mitglieder politischer Parteien und zum Teil auch Gewerkschaftsmitglieder sind. Will er etwa deren Unabhängigkeit gleichfalls in Zweifel ziehen? [...]« (FAZ vom 6. 10. 1983)

Die Verlagerung der Kontroverse über die Rolle gewerkschaftlich organisierter Richter in die Frankfurter Al]gemeine Zeitung hatte offenbar die Funktion, der Hoechst AG Zeit für neu formulierte Angriffe gegen das Frankfurter Arbeitsgericht zu gewähren. Der Vorstandsvorsitzende des Unternehmens, Prof. Dr. Rudolf Sammet, ließ dies erkennen, als er Anfang Oktober 1983 vor 1000 führenden Mitarbeitern seine Position erläuterte:

"In letzter Zeit hat der Prozeß gegen den früheren Mitarbeiter Krauß und die Tatsache, daß wir den Arbeitsrichter Feser kritisieren, für Aufsehen gesorgt ... Ich möchte aber Ihnen zu dem Fall des Arbeitsrichters Feser einige Überlegungen vortragen, die Ihnen vielleicht verständlich machen können, warum ich persönlich der Überzeugung bin, daß wir hier reagieren müssen.

Ich darf Ihnen aus dem in diesem Jahr erschienenen Handbuch des Verfassungsrechts, das von dem Präsidenten des Bundesverfassungsgerichts Benda, dem ehemaligen Innenminister Maihofer und dem ehemaligen Justizminister und heutigen Oppositionsführer Vogel herausgegeben worden ist, einen Absatz zitieren: In der jüngeren, von der Jugendbewegung von 1968 beeinflußten Richtergeneration zeigt sich gelegentlich eine Neigung, der sozialen Gerechtigkeit gegen das Gesetz unmittelbar zum Siege zu verhelfen. Die Entscheidung eines Rechtsfalles hängt dann nicht von allgemein geltenden Regeln, sondern von der sozialen Rolle von Kläger und Beklagten ab. Den Prozeß gewinnt im Zweifel der sozial Schwächere, Abhängige, Unterlegene: der Arbeitnehmer gegen den Arbeitgeber, der Mieter gegen den Vermieter, der Verbraucher gegen den Produzenten, der Kreditnehmer gegen die Sparkasse, der Versicherte gegen die Versicherungsanstalt, der Jugendliche gegen die Eltern, der Auszubildende gegen den Meister, der Schüler gegen die Schulbehörde, der Asylant gegen die Ausländerbehörde, der Demonstrant gegen den Polizeipräsidenten, der Beamtenbewerber gegen die Einstellungsbehörde.

Diese Tendenz wird mit dem Grundsatz gerechtfertigt, daß jeder gleichen Anspruch auf Freiheit hat. Es heißt, gesellschaftliche Diskriminierungen müßten durch juristische Privilegierung, gesellschaftliche Privilegien durch juristische Diskriminierung kompensiert werden ... Eine solche umgestülpte Klassenjustiz kann ihr Ziel nur über eine von Fall zu Fall - je nach der sozialen Konstellation - anders vorzunehmende Abweichung von den geltenden Rechtsregeln erreichen. Sie ersetzt die allgemeinen Gesetze durch eine Grundregel, wonach der sozial Schwächere im Zweifel den Prozeß gewinnt.

Über die Konsequenzen äußert sich der Autor wie folgt: ^Nicht nur Demokratie und Sozialstaat wären in Frage gestellt, sondern auch die Rechtssicherheit. Es gäbe kein verbindlich feststehendes Recht; weder der Bürger noch die Verwaltung wüßten, an welchen Regeln sie sich zu orientieren hätten. Gerichtliche Entscheidungen wären nur insoweit vorhersehbar, als die Kenntnis der zufälligen personellen Besetzung des zuständigen Gerichts Rückschlüsse erlaubt. Maßgebenden Einfluß hätte die publizistische Elite, die über die Medien verfügt. Sie könnte gefällige Richter loben, mißliebige Richter anprangern. Nicht nur politische Gesinnung, sondern auch menschliche Schwäche und Manipulierbarkeit der Richter könnten über den Ausgang von Rechtsstreitigkeiten entscheiden.< (Bei dem Autor, dessen Name nicht erwähnt wird, handelt es sich um Prof. Dr. Martin Kriele, R. E.)

Ich habe den Eindruck, wir haben es in unserem Fall mit einem Beispiel einer solchen rollenorientierten Rechtsprechung zu tun. Es ist ja auch ganz aufschlußreich, wenn der Direktor des Wiesbadener Arbeitsgerichts, Herr Wegener, davon spricht, daß Hoechst versuche, einen Richter - wörtliches Zitat: sabzuschießen, der die Aufgabe der Kontrolle wirtschaftlicher Macht ernst nimmtx.

Ich halte es für eine staatsbürgerliche Pflicht, diese Entwicklung nicht resignierend hinzunehmen. Wir müssen an irgendeiner Stelle auch einmal gerichtlich feststellen lassen, ob die Unabhängigkeit der Richter nicht zur Unparteilichkeit verpflichtet. Es mag sein, daß man uns sagt, daß wir da eine falsche Ansicht haben. Wir sind es aber der Allgemeinheit und auch unserer Verantwortung als Bürger dieses demokratischen Staates schuldig, eine klare Entscheidung herbeizuführen. 
Die öffentliche und veröffentlichte Meinung ist in dieser Hinsicht durchaus nicht einheitlich. Eine Reihe von Mitarbeitern hat in verschiedener Form ihre Meinung geäußert. Wir sind für dieses Engagement dankbar." (Frankfurter Allgemeine Zeitung vom IO. 10. 1983)

Was dies praktisch bedeuten sollte, erfuhr Richter Feser bald. Am I 8. 10. 1983 lehnte der Prozeßvertreter der Hoechst AG Feser erneut wegen Besorgnis der Befangenheit ab. Hatte das Unternehmen seine Ablehnungsanträge bisher damit begründet, der Richter habe zusammen mit dem Gekündigten einen Aufruf gegen den Bau der Startbahn West unterschrieben, so präsentierte es nun ein Argument, das in der mehr als einjährigen Auseinandersetzung noch nicht verwendet worden war: Richter Feser gehöre einem Arbeitskreis der ÖTV an, in dem sich Richter, Rechtsschutzsekretäre und Rechtsanwälte träfen, um rechtspolitische Fragen sowie anhängige Prozesse zu diskutieren (Handelsblatt vom 20. 10. 1983). Die Hoechst AG, der es bisher gelungen war, die Gewerkschaften weitgehend aus den Kontroversen fernzuhalten, forderte mit dieser Begründung die betroffene ÖTV zu einer Stellungnahme heraus, in der auch das Unternehmen kritisiert wurde. Der Arbeitskreis, so die Gewerkschaft, sei vor einigen Jahren eingerichtet worden, diene aber nicht dazu, anhängige Verfahren zu besprechen (Frankfurter Rundschau vom 26. 10. 1983).

$\mathrm{Zu}$ einer breiten öffentlichen Diskussion dieses Sachverhalts war es zunächst deshalb nicht gekommen, weil Richter Feser mit einer Selbstablehnung aus dem Verfahren über den Weiterbeschäftigungsanspruch des Klägers Krauss ausschied. Freilich nicht, weil er die Gründe der Hoechst AG nun akzeptierte, sondern weil er angesichts der Eskalation des Konflikts nicht mehr unbefangen entscheiden könne (Frankfurter Neue Presse vom 19. I I. 1983). Die Situation veränderte sich indes entscheidend, als die Hoechst AG den neuen Vorsitzenden, Dr. Günther Rossmanith, (vergeblich) mit dem Argument ablehnte, er habe sich nicht von einer Erklärung der Gewerkschaft ÖTV Hessen distanziert, in der diese feststellte: „Das Verhalten der Firma Farbwerke Hoechst AG (im Zusammenhang mit der Ablehnung des Arbeitsrichters Feser) ... macht deutlich, daß die Führung dieser Firma nicht gewillt ist, die vom Grundgesetz vorgegebene Ordnung der Rechtsprechung zu akzeptieren. Wenn ein mächtiger Industriebetrieb nicht einmal die Institution einer unabhängigen Rechtsprechung respektieren will, so läßt dies erahnen, wie Arbeitgeber mit den verfassungsmäßigen Rechten der Betriebsangehörigen und des Betriebsrats umspringen ... (zitiert nach Frankfurter Rundschau vom 25. I. 1984).

Als fast zur gleichen Zeit der Prozeßvertreter der Hoechst AG in der "Zeitschrift für Rechtspolitik" den engagierten Arbeitsrichtern eine "souveräne Verachtung von Art. $20 \mathrm{GG}$ « vorwarf ${ }^{\prime \prime}$, erreichte der Konflikt eine Dimension, die zu einer breiteren öffentlichen Diskussion drängte. Auf Einladung der »Humanistischen Union« fanden sich Anfang Februar mehr als 200 Personen, überwiegend Richter und Gewerkschafter, zusammen, um im Verlaufe einer Podiumsdiskussion ihrem Unmut über die Politik der Hoechst AG Ausdruck zu verschaffen (ausführlich dazu die FAZ vom 3. 2. 1984).

Wenngleich der »Fall Hoechst AG gegen Arbeitsgericht Frankfurt am Main noch nicht beendet ist ${ }^{12}$ und derzeit auch nicht vorauszusehen ist, was im Verlaufe dieser Auseinandersetzungen noch alles geschehen wird, können einige allgemeine Schlußfolgerungen gezogen werden. Der seit Ende der siebziger Jahre in der unterinstanzlichen Arbeitsgerichtsbarkeit begonnene Prozeß einer stärker sozial engagierten Rechtsprechung trifft nun - angesichts einer zum Konservatismus neigenden politi-

11 Ch. Berglar, Politischer Aktionismus in schwarzer Robe, ZRP 1984, S. ${ }_{4} \mathrm{ff}$.

12 Eine ausführliche Dokumentation des Konflikts hat die ÖTV Kreisverwaltung Frankfurt am Main (Wilhelm-Leuschner-Str. 69-77, 6000 Frankurt am Main r) nun unter dem Titel »Rechtsbeugung am Arbeitsgericht? Haben die Arbeitsgerichte Schlagseite?* herausgegeben. 
schen Öffentlichkeit - zum erstenmal auf den massiven Widerstand von Unternehmern. Diese scheinen das rechtspolitische Ziel zu verfolgen, die Entscheidungspraxis der Arbeitsgerichte zu beeinflussen, um auf diese Weise ihren tradierten »Rechten " im Betrieb wieder Geltung zu verschaffen. In diesem Bestreben können sich Unternehmen auf die veröffentlichte konservative Meinung und auf Hochschullehrer stützen, gegenüber deren "Konzertierter Aktion « die betroffenen Arbeitsrichter zwar mit Widerstand reagieren, dem persönlichen Problemdruck aber offenbar nur schwer oder gar nicht standhalten können. Entscheidendes Einfallstor für konservative Gruppen auf die erstinstanzliche Arbeitsgerichtsbarkeit ist eine politische Konstellation, in der die - Rechtsfortschritte im Arbeitsrecht maßgeblich tragenden Gewerkschaften Arbeitsrichtern keine oder nur partielle Unterstützung geben. Eine "Wende« in der erstinstanzlichen Arbeitsgerichtsbarkeit wird deshalb nur dann verhindert werden können, wenn diejenigen Institutionen, für deren Mitglieder sich eine Reihe jüngerer Arbeitsrichter engagieren, dem Unisono von Unternehmern, konservativer Presse und einigen Hochschullehrern ihre unzweideutige Ablehnung gegenüberstellen.

Rainer Erd

\section{Strafverteidigertag}

18. 5. bis 20. 5. $1984-8000$ München, Mandlstraße 23 - Kardinal-Wendel-Haus

Themen der Arbeitsgruppen:

1. POLIZEI UND STRAFPROZESS - Bestimmt die Polizei die Grenzen der Wahrheitsfindung?

Referenten: Rainer Keller, Akad. Rat, Universität Hannover; RA Uwe Maeffert, Hamburg; Falco Werkentin, Mithrsg. des Informationsdienstes »Bürgerrechte und Polizei«

2. INDIVIDUELLE UND INSTITUTIONELLE BEGEHUNG VON STRAFTATEN

DURCH POLIZEIBEAMTE; V-Mann-Probleme

Referenten: RA Dr. Sebastian Cobler, Frankfurt/Main; Albrecht Funk, Mithrsg. des Informationsdienstes »Bürgerrechte und Polizei«

3. DER KRONZEUGE - Garant der Wahrheitsfindung oder Instrument der Überführung? Referenten: Prof. Dr. Heike Jung, Universität Saarbrücken; Dr. Thomas Weigend, MaxPlanck-Institut für internationales Strafrecht, Freiburg

4. STRAFZUMESSUNG: Ideologische Nebelwand? Begründungszwang und Begründungsmanipulation

Referenten: Prof. Dr. Klaus Briegleb, Universität Hamburg; Prof. Dr. Heinz Giehring, Universität Hamburg; RA Henner Kraetsch, Berlin; Prof. Dr. Ingo Müłler, Universität Bremen; RA Reinhold Schlothauer, Bremen

5. AKTUELLE PROBLEME DES STRAFVOLLZUGS - Sondervollzug bei den Gefangenen nach $\S 129$ a StGB und dessen Auswirkung auf den Normalvollzug

Referenten: RA Erich Joester, Bremen; Denis Pecic, Strafgefangener und Mitverfasser des Alternativkomm. zum StrafvollzugsG; RA MichaeI Schubert, Freiburg

6. ALTERNATIVEN ZUR FREIHEITSSTRAFE

Referenten: Arno Pilgram, Ludwig-Boltzmann-Institut, Wien; Prof. Dr. Alexis Albrecht, Universität Bielefeld; Dr. Siegfried Lamnich, Max-Planck-Institut für internationales Strafrecht, Freiburg; Dr. Van Marle, Groningen, Holland

7. DIE GEGENREFORM MARSCHIERT - Uber die neuesten Gesetzentwürfe zur Änderung des Strafverfahrens

Referent: RA Christian Richter II, Köln

Tagungsbeitrag: DM 100,- (60,- für Studenten und Referendare) einschließlich Mittagessen am 15. 5. 1984

Anmeldung (Zimmerreservierung verbindlich nur bis 10. 4. 1984 möglich) über: Organisationsbüro 8. Straf verteidigertag, c/o RA. J. Montag, Hohenzollernstr. 102, 8000 München 40, Tel.: (089) 3081054 